\title{
Elevated Expression of Nuclear Protein Kinase CK2 $\alpha$ as a Poor Prognosis Indicator in Lymph Node Cancerous Metastases of Human Thyroid Cancers
}

\author{
Miao Guo ${ }^{1,4}$, Chao Liu', Feng-Jie $\mathbf{Q i}^{2}$, Xiu-Mei Zhang ${ }^{1}$, Li-Li Ren${ }^{1}$, Yi-Meng \\ Liu $^{1}$, Zhi-Chao Meng' ${ }^{1}$, Zhi-Tu Zhu ${ }^{3}$, Jian-Ying Xiao ${ }^{1}$
}

\begin{abstract}
Aim: To investigate the expression of protein kinase CK2 $\alpha(\mathrm{CK2} \alpha)$ in human thyroid disease and its relationship with thyroid cancer metastasis. Materials and Methods: Using immunohistochemistry we measured the expression of CK2 $\alpha$ in 76 benign and malignant human thyroid cancer tissues, including 10 pairs of papillary carcinoma tissues with or without lymph node cancerous metastasis and similarly 10 pairs of lymph nodes. Results: The expression of CK2 $\alpha$ was found to be higher in thyroid carcinoma cases (papillary carcinoma, follicular carcinoma, anaplastic carcinoma and medullary carcinoma) than in ones such as chronic lymphocytic thyroiditis, nodular goiter and adenoma. These findings were also confirmed by RT-PCR and Western blotting. More strikingly, elevated expression of $\mathrm{CK2} \alpha$ in thyroid papillary carcinoma tissues was not only significantly associated with lymph node cancerous metastasis and clinical stage of thyroid cancers; but also correlated with epithelial-mesenchymal transition (EMT) and high tenascin C (TNC) expression. In addition, EMT and high TNC expression in thyroid carcinoma tissues was significantly associated with lymph node cancerous metastasis. Conclusions: Elevated expression of nuclear $\mathrm{CK} 2 \alpha$ is a poor prognosis indicator in lymph node cancerous metastasis of human thyroid cancers.
\end{abstract}

Keywords: CK2 $\alpha$ - TNC - E-cadherin - N-cadherin - thyroid cancer

Asian Pac J Cancer Prev, 15 (17), 7425-7432

\section{Introduction}

Thyroid cancer is a common endocrine malignancy that has rapidly increased in global incidence in recent decades (Jemal et al., 2011; Howlader et al., 2012). The majority $(95 \%)$ of thyroid carcinomas derives from follicular epithelial cells and shows a spectrum of differentiation from indolent well-differentiated papillary and follicular carcinomas to more aggressive poorly differentiated carcinomas and highly lethal anaplastic carcinomas (Asa et al., 2000; Boerner et al., 2009). Medullary thyroid carcinomas (MTC) are derived from the parafollicular C-cells, they account for a minority (5\%) of thyroid carcinomas. V600E mutations in papillary thyroid carcinoma occurred more than 50\% in Iran and Indonesia (Brahma et al., 2013; Ranjbar et al., 2013). This spectrum of progression has been linked with a pattern of cumulative genetic defects that correlate with tumor differentiation, metastatic potential, and aggressiveness. Thus, thyroid cancer provides a model to examine the effects of targeted modulation of cell growth and differentiation in human neoplasms of varying degrees of differentiation and with specific genetic defects.

Protein kinase CK2 (casein kinase II) has traditionally been classified as a messenger independent protein serine/threonine kinase. It is typically found in tetrameric complexes consisting of two catalytic ( $\alpha$ and /or $\alpha^{\prime}$ ) subunits and two regulatory $\beta$ subunits (Litchfield, 2003). Protein kinase CK2 $\alpha(\mathrm{CK} 2 \alpha)$ knockout mice are not viable because of defects in heart and neural tube development (Seldin et al., 2008). It is known that CK2 $\alpha$ is involved in cell proliferation and survival (Guo et al., 1999; Buchou et al., 2003; Ahmad et al., 2008) and in several key signaling pathways such as PI3K, NF- $x$ B, Wnt and Notch1 (Guerra et al., 2006; Duncan et al., 2008; Dominguez et al., 2009; Zhang et al., 2012).

We previously reported that $\operatorname{Ser}^{149}$ is another potential protein kinase $\mathrm{A}$ (PKA) phosphorylation target of $\mathrm{Cdc} 25 \mathrm{~B}$ in $\mathrm{G} 2 / \mathrm{M}$ transition of fertilized mouse eggs (Xiao et al., 2011). In the present study, we focused on the expression pattern and prognostic significance of the CK2 $\alpha$ in thyroid cancer. Although elevated CK $2 \alpha$ expression has been found in a variety of cancers, to the best of our knowledge, the expression pattern and the prognostic significance of

${ }^{1}$ Department of Biochemical and Molecular Biology, Liaoning Medical University, ${ }^{2}$ Department of Pathology, ${ }^{3}$ Department of Oncology, ${ }^{4}$ Department of Neurosurgery, First Affiliated Hospital of Liaoning Medical University, Jinzhou, Liaoning, China *For correspondence: xiaojianying@lnmu.edu.cn 
CK2 $\alpha$ in human thyroid carcinomas are still unknown. Here, we examined the CK2 $\alpha$ expression in the tissues derived from thyroid carcinomas or begin thyroid diseases. Correlation of CK $2 \alpha$ expression with malignancy or not, with lymph node cancerous metastasis, with epithelialmesenchymal transition (EMT) and increased tenascin C (TNC) expression was performed.

\section{Materials and Methods}

\section{Patient characteristics}

We obtained paraffin-embedded samples of 47 thyroid carcinomas (including 20 with papillary carcinoma, 7 with follicular carcinoma, 9 with anaplastic carcinoma, and 11 with medullary carcinoma) and 29 other thyroid diseases (9 with chronic lymphocytic thyroiditis, 9 with nodular goiter, 11 with adenoma), that were diagnosed on the basis of histological and clinical findings at the First Affiliated Hospital of Liaoning Medical University from 2005 to 2012 . In addition, among the patients with papillary carcinoma, 10 pairs of primary thyroid tissues with or without cancerous metastasis and 10 pairs of lymph nodes with or without cancerous metastasis were included. The patients were aged $20-75$ years (mean $=49.5$ years) (Table 1). All patient samples and related data analyses were performed in accordance with research ethics approval at the Liaoning Medical University, Jinzhou, Liaoning, P. R. China.

\section{Immunohistochemistry}

Immunohistochemical staining was performed using a Dako Envision System (Dako, Carpinteria, CA, USA) following the manufacturer's recommended protocol. Briefly, all paraffin sections, $4 \mu \mathrm{m}$ in thickness, were heated for 2 hours (h) at $60^{\circ} \mathrm{C}$, deparaffinized with xylene, rehydrated through a graded series of ethanol/ distilled water, submerged in EDTA buffer ( $\mathrm{pH} 9.0$ or $\mathrm{pH}$ 6.0), heated in a microwave for antigen retrieval, treated with $3 \% \mathrm{H} 2 \mathrm{O} 2$ for 15 minutes (min) to block the endogenous peroxidase, incubated with primary antibody at $4^{\circ} \mathrm{C}$ overnight. The primary reactions were as follows: anti-Casein kinase II (alpha subunit) rabbit monoclonal antibody (at 1:80 dilution, Millipore, Darmstadt, Germany), anti-E-cadherin (working solution, Beijing zhongshanjinqiao Biotechnology Co. Ltd, Beijing, P. R. China), anti-N-cadherin (working solution, Beijing zhongshanjinqiao Biotechnology Co. Ltd) and antiTenascin C (at 1:100 dilution, Abcam, Cambridge United Kingdom) were used. The sections were incubated with horseradish perfidies (HRP) at $37^{\circ} \mathrm{C}$ for $30 \mathrm{~min}$, and visualized with diaminobenzidine (DAB). For negative controls, the antibody was replaced by PBS.

\section{Evaluation of staining}

All digital images were captured, in a double-blind manner, from five random fields per section using an Olympus microscope. The images were taken at $40 \mathrm{X}$ magnification for $\mathrm{CK} 2 \alpha$, E-cadherin, $\mathrm{N}$-cadherin and Tenascin $\mathrm{C}$ positive cell counting. We counted digital images of CK2 $\alpha$, E-cadherin, N-cadherin and Tenascin $\mathrm{C}$ positive cells separately by two pathologists who were
Table 1. Clinicopathological Characteristics of the 76 Patients

\begin{tabular}{lr}
\hline & $\mathrm{N}(\%)$ \\
\hline Gender & \\
Male & $19(25)$ \\
Female & $57(75)$ \\
Age & \\
$\geq 45$ & $29(38.2)$ \\
$<45$ & $47(61.8)$ \\
Pathological type & \\
Chronic lymphocytic thyroiditis & $9(11.8)$ \\
Nodular goiter & $9(11.8)$ \\
Adenoma & $11(14.5)$ \\
Papillary carcinoma & $20(26.4)$ \\
Follicular carcinoma & $7(9.2)$ \\
Anaplastic carcinoma & $9(11.8)$ \\
Medullary carcinoma & $11(14.5)$ \\
Degree of differentiation & \\
Well & $56(73.7)$ \\
Moderately & $11(14.5)$ \\
Poorly & $9(11.8)$ \\
TNM Stage & \\
I-II & 24 \\
III-IV & 23 \\
\hline
\end{tabular}

Table 2. Correlation between the clinicopathological features and expression of the CK2 $2 \alpha$ protein in thyroid carcinomas

\begin{tabular}{lccc}
\hline & $\mathrm{N}$ & $\mathrm{CK} 2 \alpha(\%)$ & $p$ \\
\hline Gender & & & 0.386 \\
$\quad$ Male & 19 & 42.09 & \\
$\quad$ Female & 57 & 50.03 & \\
Age & & & 0.624 \\
$\quad 245$ & 29 & 39.52 & \\
$\quad<45$ & 47 & 49.58 & \\
Degree of differentiation & & & 0.052 \\
$\quad$ Well & 27 & 38.87 & \\
$\quad$ Moderately & 11 & 45.65 & \\
$\quad$ Poorly & 9 & 48.77 & \\
TNM Stage & & & $<0.001$ \\
$\quad$ I-II & 24 & 37.36 & \\
$\quad$ III-IV & 23 & 59.87 & \\
\hline
\end{tabular}

blinded to the clinical parameters. Values from five random fields were averaged to a single value per case. The results were expressed as percentage of the number of CK $2 \alpha$, E-cadherin and N-cadherin positive cells/total cells.

\section{RNA extraction, cDNA Synthesis and PCR}

Total RNA from 4 tumor and non-tumor pairs of thyroid tissues was isolated by Trizol (Invitrogen, Camarillo, CA, USA), according to the manufacturer's instructions and cDNA was prepared from $1 \mu \mathrm{g}$ total RNA in a $20 \mu \mathrm{l}$ volume using RNA PCR Kit (AMV) Ver 3.0 (Takara, Shiga, Japan). The reaction mixture was incubated at $42^{\circ} \mathrm{C}$ for $30 \mathrm{~min} ; 99^{\circ} \mathrm{C}$ for $5 \mathrm{~min} ; 5^{\circ} \mathrm{C}$ for $5 \mathrm{~min}$. The synthesized cDNA was used for PCR amplification or stored at $-20^{\circ} \mathrm{C}$ for further analysis.

The PCR reaction was performed in a $25 \mu \mathrm{l}$ volume containing $0.25 \mathrm{mM}$ of each dNTP (Takara), 1 U Hotstar Taq (Takara) and $0.5 \mathrm{mM}$ each Primer (Takara) 5' - CCGAGTTGCTTCCCGATAC -3' and 
5'- GGGCTGACAAGGTGCTGAT -3' (for CK2 $\alpha$ ) , 5'-CTTTGGTATCGTGGAAGGACTC-3' and 5'-GTAGAGGCAGGGATGATGTTCT-3' (for GAPDH). Reaction conditions included initial denaturation at $95^{\circ} \mathrm{C}$ for $10 \mathrm{~min}$, followed by 35 cycles at $95^{\circ} \mathrm{C}$ for 1 min, annealing at $56^{\circ} \mathrm{C}$ for $1 \mathrm{~min}$ and extension at $72^{\circ} \mathrm{C}$ for $1 \mathrm{~min}$, followed by a $10 \mathrm{~min}$ final extension at $72^{\circ} \mathrm{C}$. PCR products were separated on $3.0 \%$ agarose gels and visualized by ethidium bromide staining.

\section{Protein isolation and Western blotting}

Four tumor and non-tumor pairs of thyroid tissues were homogenized using a poly-tron homogenizer in radioimmunoprecipitation assay (RIPA) lysis buffer (1x PBS, $1 \%$ Nonidet P-40, $0.5 \%$ sodium deoxycholate, $0.1 \%$ SDS, ) with protease inhibitor cocktail tablet (Roche, Basel, Swiss)

Equal amounts of protein $(50 \mu \mathrm{g})$ solubilized in sample buffer were separated on $10 \%$ SDS polyacrylamide gels and transferred electrophoretically onto nitrocellulose membranes. Membranes were blocked in Tris-buffered saline containing $0.1 \%$ Tween 20 (TBS-T) plus 5\% nonfat dried milk for $1 \mathrm{~h}$ at room temperature, probed with primary CK2 $\alpha$ (1:1000 dilution, Millipore) and actin (1:1000 dilution, Beijing zhongshanjinqiao Biotechnology Co. Ltd) antibodies at $4^{\circ} \mathrm{C}$ overnight. The membranes were washed three times for 10-15 min each time in TBS-T, and incubated with horseradish peroxidase-conjugated goat anti-rabbit secondary antibody (1:2000 dilution, Beijing zhongshanjinqiao Biotechnology Co. Ltd) for $1 \mathrm{~h}$ at room temperature. Targeted proteins were visualized using an ECL chemiluminescence detection system (Beyotime Institute of Biotechnology, Beijing P. R. China). Gel Pro analysis software Image J software (developed at the National Institute of Health, Bethesda, MD) to quantify the amount of protein. Densitometric values were normalized to those of Actin.

\section{Statistical analysis}

Data are presented as mean $(\bar{\chi}), 95 \%$ confidence interval $(\mathrm{CI})$ and $p$ value. Statistical analysis was done using the Welch t test, ONE WAY ANOVA, $\chi^{2}$ test, Spearman correlation and Mann-Whitney U-test by SPSS 13.0. P values of 0.05 or less were considered statistically significant.

\section{Results}

Increased nuclear CK2 $\alpha$ expression in thyroid carcinomas was associated with lymph node cancerous metastasis

In the present study, nuclear CK2 $\alpha$ expression was analyzed in 76 patients with thyroid carcinomas and benign diseases using immonohistochemistry. The results showed that the nuclear CK2 $\alpha$ expression was significantly increased in thyroid carcinomas $(n=47,=47.84,95 \%$ $\mathrm{CI}=44.41$ to 51.97 ) compared to its expression in benign thyroid diseases $(n=29,=10.65,95 \% \mathrm{CI}=9.41$ to 12.03 , $p<0.001)$. The staining for nuclear CK2 $\alpha$ was much weaker in 29 cases with benign thyroid diseases, including chronic lymphocytic thyroiditis $(10.32 \%)$, nodular goiter $(10.52 \%)$ and adenoma (11.06\%). However, it was much stronger expressed with a nuclear localization in 47 thyroid malignancies, including papillary thyroid carcinoma $(41.33 \%)$, follicular carcinoma (47.26\%), anaplastic carcinoma (63.68\%) and medullary carcinoma (54.02\%) (Figure 1A). Nuclear CK2 $\alpha$ expression was significantly elevated in anaplastic $(n=9,=63.68,95 \%$ $\mathrm{CI}=50.28$ to $77.07, p<0.001)$ and medullary carcinomas $(\mathrm{n}=11,=54.02,95 \% \mathrm{CI}=44.31$ to $63.73, p=0.016)$ compared to in the papillary carcinoma $(n=20,=41.33$, 95\% CI=32.36 to 50.39); and increased in the anaplastic carcinoma compared to the one in follicular carcinoma $(\mathrm{n}=7,=47.26,95 \% \mathrm{CI}=41.12$ to $53.39, p=0.017)$. To validate the immunohistochemical results, the gene and

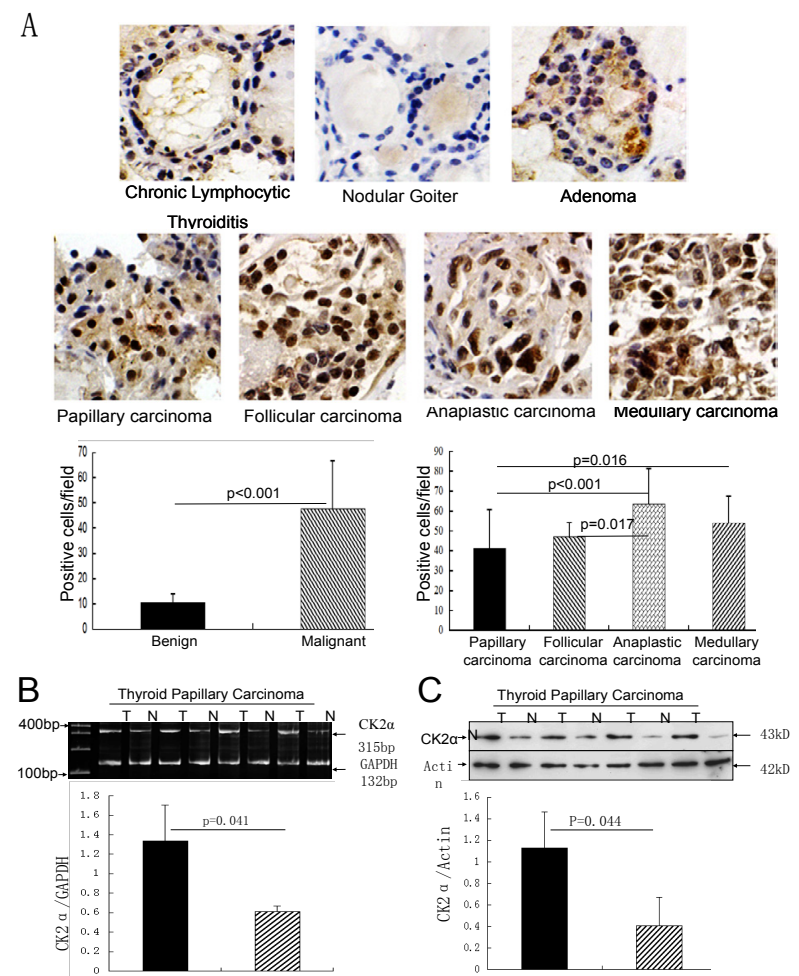

Figure 1. Expression of CK2 $\alpha$ was Elevated in Thyroid Carcinomas. (A) CK2 $\alpha$ expression in thyroid benign and malignant tissues was analyzed using immunohistochemistry. Elevated nuclear expression of CK2 $\alpha$ was found in thyroid carcinomas $(n=47,=47.84,95 \% \mathrm{CI}=44.41$ to 51.97$)$ compared to thyroid benign diseases $(\mathrm{n}=29,=10.65,95 \% \mathrm{CI}=9.41$ to 12.03 , $p<0.001)$. Higher nuclear expression of CK2 $\alpha$ was also observed in anaplastic $(\mathrm{n}=9,=63.68,95 \% \mathrm{CI}=50.28$ to $77.07, p<0.001)$ and medullary carcinomas $(\mathrm{n}=11,=54.02,95 \% \mathrm{CI}=44.31$ to $63.73, p=0.016)$ than in papillary carcinoma $(n=20,=41.33$, $95 \% \mathrm{CI}=32.36$ to 50.39 ); and increased in anaplastic carcinoma compared to follicular carcinoma $(\mathrm{n}=7,=47.26,95 \% \mathrm{CI}=41.12$ to $53.39, p=0.017$ ). (B) CK2 $\alpha$ expression at mRNA levels in thyroid benign and malignant tissues was also analyzed using RT-PCR. Four pairs of normal and papillary carcinoma tissues were chosen for total RNA extraction and RT-PCR. The results revealed that higher levels of CK $2 \alpha$ mRNA were detected in the carcinoma tissues than in the normal ones $(p=0.041)$. GAPDH was as a housekeeping gene used for CK $2 \alpha$ level normalization. (C) CK2 $\alpha$ expression at protein levels in thyroid benign and malignant tissues was also analyzed using Western blotting. Four pairs of normal and papillary carcinoma tissues (same as used for RT-PCR) were chosen for protein extraction. Western blotting showed that higher levels of CK $2 \alpha$ protein were detected in the carcinoma tissues than in the normal ones $(p=0.044)$. Actin was used for CK2 $\alpha$ level normalization 
protein levels of CK2 $\alpha$ expression were also examined in 4 pairs of normal-papillary thyroid carcinoma tissues using RT-PCR and western blotting respectively (Figure $1 \mathrm{~B}$ and $\mathrm{C}$ ). To further explore the role of $\mathrm{CK} 2 \alpha$ in thyroid cancers, we investigated the association of CK $2 \alpha$ expression with the clinicopathological characteristics of thyroid carcinoma cases and found that the expression of the CK2 $\alpha$ protein in thyroid carcinomas at the IIIIV stage was significantly higher than in the I-II stage $(p<0.001)$. However, no significant correlation was found between CK $2 \alpha$ expression and gender, age, degree of differentiation $(p>0.05)$ (Table 2).

More strikingly, nuclear CK2 $\alpha$ expression was significantly elevated in papillary thyroid carcinoma tissues with lymph node cancerous metastasis $(n=10$, $=54.25,95 \% \mathrm{CI}=46.07$ to 63.23 ) compared to its expression in papillary thyroid carcinoma tissues without lymph node cancerous metastasis $(n=10,=28.4,95 \%$ $\mathrm{CI}=18.75$ to $38.09, p=0.003$, Figure $2 \mathrm{~A}$ ). In addition, the immunohistochemistry showed that the expression of CK $2 \alpha$ was detected in cancerous metastatic region of metastatic lymph nodes of 9 of 10 patients, but, not in un-metastatic lymph nodes of 10 cases $(p<0.001)$. The cells with $\mathrm{CK} 2 \alpha$ positive immunostaining were metastatic thyroid cancer cells (Figure 2B).

Decreased expression of E-cadherin and increased expression of $\mathrm{N}$-cadherin in thyroid carcinomas was associated with lymph node cancerous metastasis

EMT is closely linked to conversion of early-stage tumors into invasive malignancies. To investigate the mechanisms of CK $2 \alpha$ roles in thyroid carcinomas, particularly whether elevated CK2 $\alpha$ expression correlated with EMT, we analyzed E-cadherin and N-cadherin expression in human thyroid tissues.
A

B

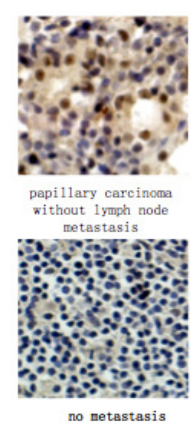

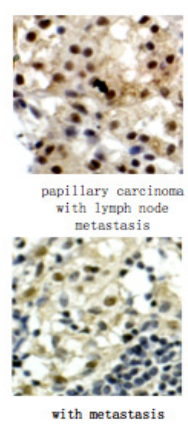

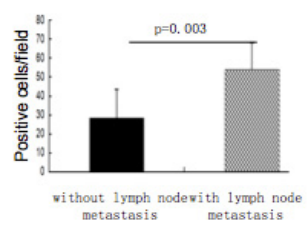

Figure 2. Elevated expression of $\mathrm{CK} 2 \alpha$ in Thyroid Carcinomas was Associated with lymph Node Cancerous Metastasis. (A) Immunohistochemistry demonstrated that nuclear expression of CK $2 \alpha$ was increased in thyroid papillary carcinoma tissues with lymph node cancerous metastasis ( $\mathrm{n}=10,=54.25,95 \% \mathrm{CI}=46.07$ to 63.23$)$ compared to in the ones without lymph node cancerous metastasis $(\mathrm{n}=10,=28.4$, 95\% $\mathrm{CI}=18.75$ to $38.09, p=0.003$ ). Increased CK2 $\alpha$ expression in papillary carcinoma was significantly associated with lymph node cancerous metastasis. (B) CK2 $\alpha$ expression in lymph node tissues was also analyzed using immunohistochemistry. Nonmetastatic lymph nodes of 10 papillary carcinoma cases did not show any immunostaining for CK2 $\alpha$. But, 9 of 10 cancerous metastatic lymph nodes showed positive staining for CK2 $\alpha$ $(p<0.001)$. The CK $2 \alpha$ positive staining cells in lymph nodes were metastatic thyroid cancer cells.
In the present study, we found that decreased E-cadherin expression was observed in thyroid carcinomas $(\mathrm{n}=47,=29.93,95 \% \mathrm{CI}=21.38$ to 39.26$)$ compared to its expression in benign thyroid diseases $(\mathrm{n}=29,=89.56,95 \%$ $\mathrm{CI}=87.42$ to $91.39, p<0.001)$. The staining for E-cadherin was stronger in 29 cases with benign thyroid diseases, including chronic lymphocytic thyroiditis $(87.19 \%)$, nodular goiter (91.15\%) and adenoma (90.24\%). However, its staining was much weaker in 47 thyroid malignancies, including papillary carcinoma $(55.61 \%)$, follicular carcinoma $(22.08 \%)$, anaplastic carcinoma $(7.39 \%)$ and medullary carcinoma (4.92\%) (Figure 3A). In addition, E-cadherin expression was increased in papillary carcinoma $(n=20,=55.61,95 \% \quad C I=43.84$ to 67.38$)$ compared to in follicular $(\mathrm{n}=7,=22.08,95 \% \mathrm{CI}=6.04$ to $38.13, p<0.001)$, anaplastic $(\mathrm{n}=9,=7.39,95 \% \mathrm{CI}=0.76$ to $15.53, p<0.001)$ and medullary carcinomas $(\mathrm{n}=11,=4.92$, $95 \% \mathrm{CI}=0.59$ to $9.24, p<0.001$ ). Higher expression of E-cadherin was also found in follicular carcinoma than in anaplastic $(p=0.05)$ and medullary carcinomas $(p=0.016)$. This pattern of E-cadherin expression in thyroid cancers is consistent with what we expected, namely lower expression of E-cadherin is associated with higher degree of malignancy.

In papillary thyroid carcinoma E-cadherin expression was higher in the thyroid tissues without lymph node cancerous metastasis $(n=10,=72.28,95 \% C I=61.97$ to 84.28 ) than with lymph node cancerous metastasis $(\mathrm{n}=10,=38.94,95 \% \mathrm{CI}=30.30$ to $46.22, p=0.007$, Figure 3 B). Down-regulated expression of E-cadherin was significantly associated with lymph node cancerous metastasis. In addition, the immunostaining showed that the expression of E-cardherin in cancerous metastatic region of metastatic lymph nodes of 6 of 9 CK $2 \alpha$ positive patients, but not in un-metastatic lymph nodes (0 of 9 cases, $p<0.001$, Figure $3 \mathrm{C}$ ). The cells with E-cadherin positive immunostaining were metastatic thyroid cancer cells.

$\mathrm{N}$-cadherin expression was much higher in thyroid carcinomas $(n=47,=42.74,95 \% \mathrm{CI}=36.85$ to 48.94$)$ than in benign thyroid diseases $(\mathrm{n}=29,=0, p<0.001$, Figure $4 A$ ). There was no staining for $\mathrm{N}$-cadherin in 29 cases with benign thyroid diseases, including chronic lymphocytic thyroiditis, nodular goiter and adenoma. However, it was very strongly expressed with 47 thyroid malignancies, including papillary thyroid carcinoma $(31.89 \%)$, follicular carcinoma (42.68\%), anaplastic carcinoma $43.6 \%)$ and medullary carcinoma $(57.89 \%)$. N-cadherin was lower expressed in papillary carcinoma $(n=20,=31.89,95 \%$ $\mathrm{CI}=19.87$ to 43.91$)$ than in medullary carcinoma $(\mathrm{n}=11$, $=57.89,95 \% \mathrm{CI}=45.5$ to $70.28, p<0.001$ ), but not in follicular and anaplastic carcinomas.

$\mathrm{N}$-cadherin expression was more increased in papillary thyroid carcinoma tissues with lymph node cancerous metastasis $(\mathrm{n}=10,=49.9,95 \% \mathrm{CI}=35.48$ to 62.88$)$ than in papillary thyroid carcinoma tissues without lymph node cancerous metastasis $(n=10,=13.88,95 \% \mathrm{CI}=10.03$ to $17.61, p=0.002$, Figure 4B). Up-regulated expression of $\mathrm{N}$-cadherin was significantly associated with lymph node cancerous metastasis. In addition, the immunostaining showed that the expression of $\mathrm{N}$-cadherin was only 


\section{A}

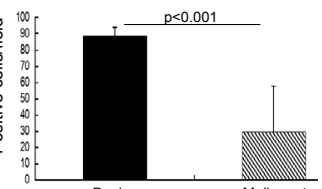

B

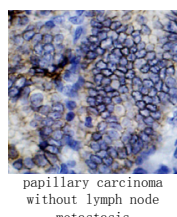

C
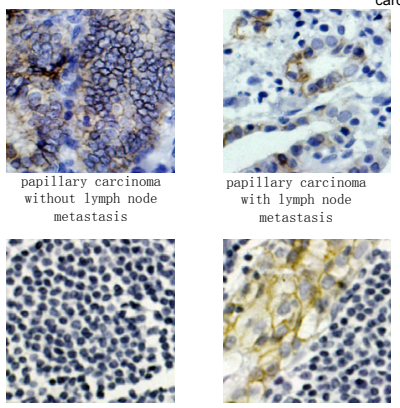
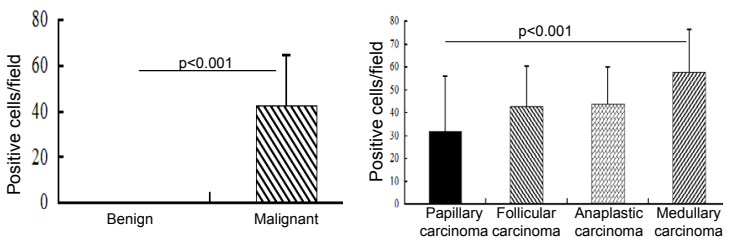

$\mathrm{B}$
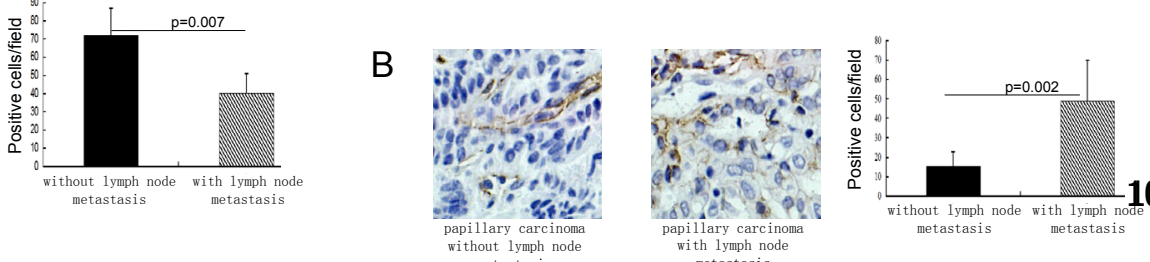

C

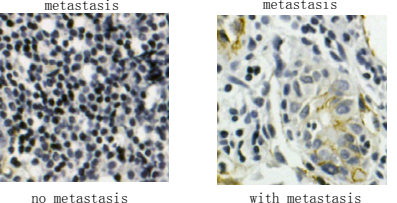

Figure 3. Decreased E-cadherin Expression in Thyroid Carcinomas was Significantly Associated with Lymph Node Cancerous Metastasis. (A) E-cadherin expression in thyroid benign and malignant tissues was analyzed using immunohistochemistry. The results showed that decreased E-cadherin expression was found in 47 thyroid carcinoma tissues $(n=47,=29.93,95 \% \mathrm{CI}=21.38$ to 39.26 ) compared to its expression in 29 thyroid benign ones $(n=29,=89.56,95 \%$ $\mathrm{CI}=87.42$ to $91.39, p<0.001)$. Among the malignant carcinomas, papillary carcinoma $(n=20,=55.61,95 \% C I=43.84$ to 67.38$)$ showed increased E-cadherin expression compared to follicular $(\mathrm{n}=7,=22.08,95 \% \mathrm{CI}=6.04$ to $38.13, p<0.001)$, anaplastic $(\mathrm{n}=9,=7.39,95 \% \mathrm{CI}=0.76$ to $15.53, p<0.001)$ and medullary carcinomas $(n=11,=4.92,95 \% \mathrm{CI}=0.59$ to $9.24, p<0.001)$. Higher E-cadheirn expression was also found in follicular carcinoma than in anaplastic $(p=0.05)$ and medullary carcinomas $(p=0.016)$. (B) Immunohistochemistry demonstrated that expression of E-cadherin was decreased in thyroid papillary carcinoma tissues with lymph node cancerous metastasis $(n=10,=38.94$, $95 \% \mathrm{CI}=30.30$ to 46.22 ) compared to its expression in the ones without lymph node cancerous metastasis $(\mathrm{n}=10,=72.28,95 \%$ $\mathrm{CI}=61.97$ to $84.28, p=0.007)$. Decreased E-cadherin expression in papillary carcinoma was significantly associated with lymph node cancerous metastasis. (C) E-Cadherin expression in lymph node tissues was also analyzed using immunohistochemistry. Non-metastatic lymph nodes (0 of 9 cases) did not show any immunostaining for E-cadherin, but, 6 of 9 CK $2 \alpha$ positive cancerous metastatic lymph nodes showed positive staining for E-cadherin $(p<0.001)$. The E-cadherin positive staining cells in lymph nodes were metastatic thyroid cancer cells

detected in thyroid cancerous metastatic region of metastatic lymph nodes in 7 of 9 nuclear CK2 $\alpha$ positive patients, not in un-metastatic lymph nodes $(0$ of 9 cases, $p<0.001$, Figure $4 \mathrm{C})$. The cells with $\mathrm{N}$-cadherin positive immunostaining were metastatic thyroid cancer cells.

High expression of TNC in thyroid carcinomas was associated with lymph node cancerous metastasis

First, we investigated the expression of TNC in primary tissues of thyroid carcinomas and benign thyroid diseases. Dense TNC deposition was only observed in areas of human thyroid carcinomas (39 of 47 cases, Figure $5 \mathrm{~A})$, whereas the tissues of benign thyroid diseases were negative for TNC (0 of 29 cases, not shown, $p<0.001$ ). TNC staining was dominantly positive in the stroma around and between the cancer cells. More strikingly, TNC was expressed more frequently in papillary thyroid

Figure 4. Increased N-cadherin Expression in Thyroid Carcinomas was Significantly Associated with lymph Node Cancerous Metastasis. (A) N-cadherin expression in thyroid benign and malignant tissues was analyzed using immunohistochemistry. The results showed that increased $\mathrm{N}$-cadherin expression was found in thyroid carcinoma tissues $(\mathrm{n}=47,=42.74,95 \% \mathrm{CI}=36.85$ to 48.94$)$ compared to its expression in thyroid benign ones $(\mathrm{n}=29,=0, p<0.001)$. Among the malignant carcinomas, medullary carcinoma $(n=11,=57.89$, 95\% $\mathrm{CI}=45.5$ to 70.28 ) showed high $\mathrm{N}$-cadherin expression compared to papillary carcinoma $(\mathrm{n}=20,=31.89,95 \% \mathrm{CI}=19.87$ to $43.91, p<0.001$ ). (B) Immunohistochemistry revealed that expression of $\mathrm{N}$-cadherin was increased in thyroid papillary carcinoma tissues with lymph node cancerous metastasis $(\mathrm{n}=10$, $=49.9,95 \% \mathrm{CI}=35.48$ to 62.88$)$ compared to its expression in the ones without lymph node cancerous metastasis $(\mathrm{n}=10$, $=13.88,95 \% \mathrm{CI}=10.03$ to $17.61, p=0.002$ ). Increased $\mathrm{N}$-cadherin expression in papillary carcinoma was significantly associated with lymph node cancerous metastasis. (C) N-cadherin expression in lymph node tissues was also analyzed using immunohistochemistry. Non-metastatic lymph nodes (0 of 9 cases) did not show any immunostaining for $\mathrm{N}$-cadherin, but, 7 of 9 CK $2 \alpha$ positive cancerous metastatic lymph nodes showed positive staining for $\mathrm{N}$-cadherin $(p<0.001)$. The $\mathrm{N}$-cadherin positive staining cells in lymph nodes were metastatic thyroid cancer cells

carcinoma tissues with lymph node cancerous metastasis (10 of 10 cases) than in papillary thyroid carcinoma tissues without lymph node cancerous metastasis (4 of 10 cases, $p=0.011$, Figure 5B). Up-regulated expression of TNC was significantly associated with lymph node cancerous metastasis.

In addition, the immunhistochemistry showed that the expression of TNC in cancerous metastatic lymph nodes of 5 of 9 nuclear CK $2 \alpha$ positive patients, but not in unmetastatic lymph nodes of 9 cases (Figure 5C). There was significant difference between these 2 groups $(p=0.029)$. The increased expression of TNC in lymph nodes of papillary thyroid carcinoma is significant associated with lymph node cancerous metastasis.

Increased expression of nuclear CK2 $\alpha$ in thyroid carcinoma correlated with EMT and increased TNC expression

Although there are many papers reporting elevated 
Miao Guo et al

\section{A}
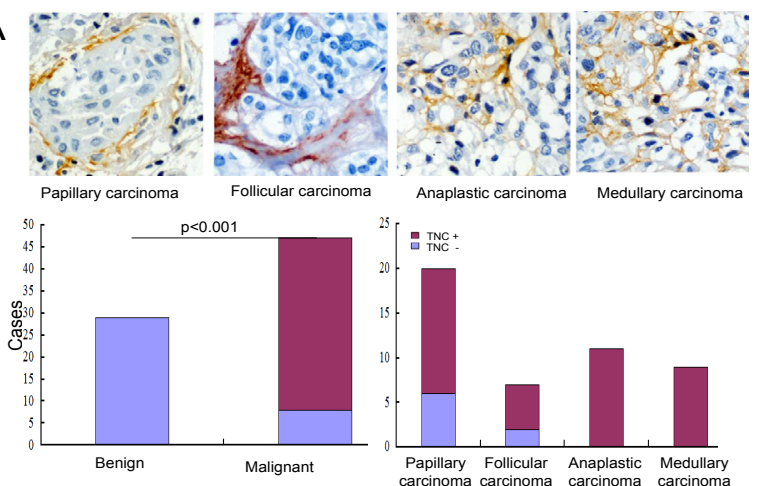

B
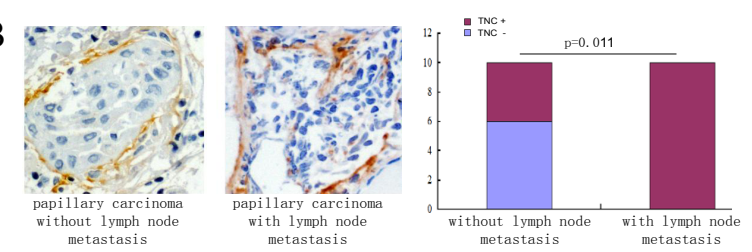

C
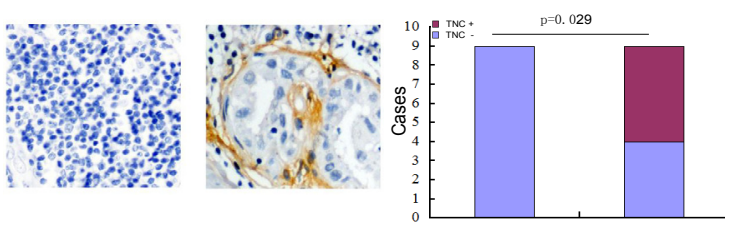

Figure 5. Dense TNC Deposition Correlated with Human Thyroid Malignancy and Lymph Node Cancerous Metastasis. (A) TNC staining was frequently positive in the stroma with scattered cancer cells in thyroid carcinoma tissues (39 of 47 cases). However, the tissues of human benign thyroid diseases did not show any staining for TNC ( 0 of 29 cases, not shown). The difference in positivity of TNC staining between thyroid carcinomas and benign diseases was significant $(p<0.001)$. Among the malignant carcinomas there was no any significant difference each other. (B) TNC staining was positive in all thyroid papillary carcinoma tissues with lymph node cancerous metastasis ( 10 of 10 cases). However, only 4 of 10 cases without lymph node cancerous metastasis showed TNC positive staining. There is significant difference between these two groups $(p=0.011)$. (C) TNC staining were significantly more often found in the lymph node tissues with cancerous metastasis (5 of 9 cases) than in the ones without cancerous metastasis ( 0 of 9 cases, $p=0.029$ )

CK2 $\alpha$ in some types of cancers, there is no paper about the correlation of its expression with the EMT and TNC expression in thyroid cancers. By statistical analysis we already found that high expression of CK2 $\alpha$ in primary thyroid cancer tissues was positively associated with cancerous lymph node metastasis (Figure 2A), which supports CK2 $\alpha$ acts as a cancer driver to promote cancer cell invasion and metastasis in addition to stimulating tumorigenesis. Consistently with these effects, elevated CK2 $\alpha$ expression in thyroid cancers negatively correlated with decreased E-cadherin (Figure 6A, rho $=-0.75$, $p<0.001$ ), positively with increased $\mathrm{N}$-cadherin expression (Figure 6B, rho $=0.757, p<0.001$ ) and the number of TNC positive is increased with the increased expression of CK2 $\alpha(p<0.001$, Figure 6C). From these findings we could conclude that $\mathrm{CK} 2 \alpha$ expression is significantly more increased in thyroid carcinomas than in normal thyroid diseases; and elevated expression of CK2 $\alpha$ in papillary carcinomas is significantly associated with lymph node
A

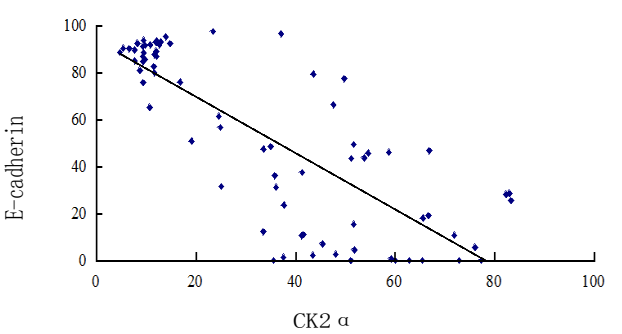

B

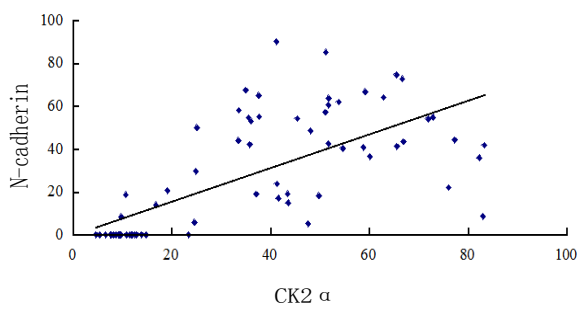

C

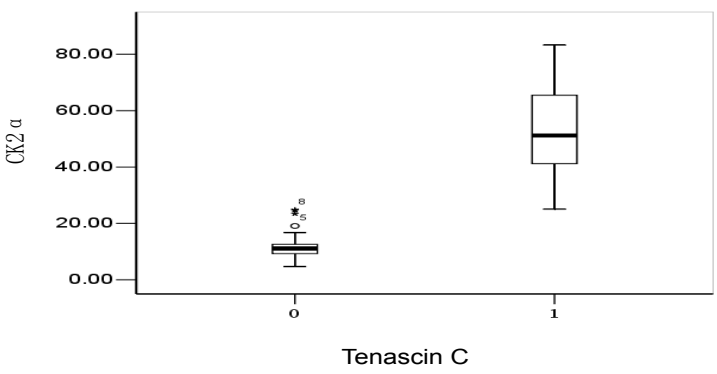

Figure 6. Elevated Expression of CK2 $\alpha$ in Thyroid Carcinomas was Correlated with Induction of EMT and Increased TNC Expression. From the above results, we knew that the differential expression of CK2 $\alpha$, E-cadherin, $\mathrm{N}$-cadherin and TNC was respectively associated with lymph node cancerous metastasis. The Spearman correlation showed that elevated expression of CK2 $\alpha$ was significantly correlated with decreased E-cadherin (A), increased N-cadherin (B) in primary thyroid papillary carcinoma tissues; and the number of TNC positive is increased with the increased expression of CK2 $\alpha(\mathrm{C})$

cancerous metastasis and correlated with induction of EMT and increased TNC expression. Induction of EMT and elevated TNC expression is at least a partial mechanism of CK $2 \alpha$ roles in thyroid cancer development, invasion or metastasis.

\section{Discussion}

Elevated CK2 $\alpha$ expression has been reported in some different kind of cancers including mammary gland (Landesman-Bollag et al., 2001), head and neck (Faust et al., 1996), kidney cancers (Stalter et al., 1994). Elevated level and prognostic significance of CK $2 \alpha$ have been found in colorectal cancer (Pornchai et al., 2004), lung cancer (Lin et al., 2011), prostate cancer (Laramas et al., 2007) and leukemia (Kim et al., 2007). These findings support the idea that $\mathrm{CK} 2 \alpha$ acts as a cancer driver. However, the role of CK $2 \alpha$ in cancer development, progression and metastasis has yet to be fully elucidated. To date, there is no report about the expression pattern and potential implications of CK $2 \alpha$ in thyroid carcinomas.

In the present study, the nuclear expression of CK2 $\alpha$ in thyroid tissues derived from 76 patients with 
benign and malignant diseases was assessed. Using immunohistochemistry we demonstrated for the first time that nuclear CK2 $\alpha$ expression was significantly increased in thyroid cancer tissues compared to its expression in benign diseases. Interestingly, the expression of the CK2 $\alpha$ protein in thyroid carcinomas at the III-IV stage was significantly higher than in the I-II stage and the CK2 $\alpha$ expression was positively associated with the level of malignancy of thyroid carcinomas indicating that CK $2 \alpha$ levels are associated with thyroid cancer development and progression. More strikingly, the elevated nuclear CK2 $\alpha$ expression was significantly associated with lymph node cancerous metastasis. These findings support that CK $2 \alpha$ plays an important role not only as a cancer driver but also as a metastatic indicator in thyroid cancers. Consistent with these findings it has been recently reported that CK $2 \alpha$ over-expression correlated with metastatic risk in breast carcinomas (Giusiano et al., 2011).

However, the mechanisms for the correlation of CK $2 \alpha$ expression with cancerous metastasis remain unclear. EMT is a process known to contribute to invasion and metastasis in cancer and it is mainly characterized by loss of cell polarity and a change in cell shape from cuboidal to fibroblastoid, the downregulation of epithelial markers and the upregulation of mesenchymal markers. The cell acquires the capacity to degrade the basement membrane and migrate through the extracellular matrix to populate different territories either during embryonic development or cancer progression (Acloque et al., 2009). The switch in expression from E-cadherin to N-cadherin is used to monitor EMT during cancer progression. In the current study, we found decreased E-cadherin and increased $\mathrm{N}$-cadherin expression in thyroid carcinomas compared to thyroid benign diseases. Importantly, the elevated expression of nuclear CK $2 \alpha$ was significantly correlated with EMT in thyroid cancers. This novel finding might represent a novel mechanistic part of CK $2 \alpha$ involvement in thyroid cancer development, progression and metastasis.

TNC is an extracellular matrix glycoprotein that exhibits de-adhesive effects on cell-matrix interaction. TNC is highly expressed in remodeling tissues during embryonic development and is frequently up-regulated in a variety of pathological conditions including chronic inflammation and cancers (Midwood et al., 2011). TNC is early described as a stormal marker associated with malignant behavior of breast cancer (Mackie et al., 1987). In addition, TNC is highly expressed in brain tumor (Leins et al., 2003), head and neck cancers (Lyons et al., 2007; Atula et al., 2003). In the above described tumors, the high expression TNC is associated with invasion and worse prognosis. In lung and primary breast tumor tissues high expression of TNC is associated with progression to lung replase (Oskarsson et al., 2011). It was reported that TNC was expressed in most papillary and medullary carcinomas and C-cell hyperplasia (Tseleni-Balafouta et al., 2006; Koperek et al., 2009). In the current study, we found that TNC was also expressed in thyroid follicular and anaplastic carcinomas in addition to papillary and medullary carcinomas. More importantly, we demonstrated for the first time that elevated TNC expression in thyroid papillary carcinoma was significantly associated with lymph node cancerous metastasis; and cancerous metastatic lymph nodes showed higher TNC staining compared to non-cancerous metastatic lymph nodes.

In summary, our findings uncover the potential role of CK $2 \alpha$ in thyroid cancer development, progression and metastasis. The expression of nuclear CK $2 \alpha$ was increased in thyroid carcinomas compared to benign thyroid diseases. The elevated expression of CK2 $\alpha$ was significantly associated with lymph node cancerous metastasis and clinical stage of thyroid cancers, which might be induced by EMT and increased expression of TNC in thyroid cancers. From this study we concluded that elevated expression of nuclear CK2 $\alpha$ is a poor prognostic indicator in lymph node cancerous metastasis of human thyroid cancers.

We first found the expression of CK2 $\alpha$ is higher in carcinoma tissues and elevated expression of CK $2 \alpha$ in thyroid papillary carcinoma tissues was not only significantly associated with lymph node cancerous metastasis but also correlated with epithelial-mesenchymal transition (EMT) and high tenascin C (TNC) expression. Elevated expression of CK2 $\alpha$ was also significantly associated with clinical stage of the patients with thyroid cancers. In addition, EMT and high TNC expression in thyroid carcinoma tissues was significantly associated with lymph node cancerous metastasis. So elevated expression of nuclear protein kinase $\mathrm{CK} 2 \alpha$ as a poor prognostic indicator in lymph node cancerous metastasis of human thyroid cancers

\section{Acknowledgements}

The work was supported by a research grant from foundation of Technology Department of Liaoning Province of China (2013225305).

\section{References}

Acloque H, Adams MS, ONE MORE, et al (2009). Epithelialmesenchymal transitions: the importance of changing cell state in development and disease. J Clin Invest, 119, 1438-49.

Ahmad KA, Wang G, ONE MORE, et al (2008). Protein kinase CK2--a key suppressor of apoptosis. Adv Enzyme Regul, 48, 179-87.

Asa SL, Bedard YC, (2000). Fine-needle aspiration cytology and histopathology. InClark OH, Noguchi S (eds.). Thyroid cancer. Diagnosis and treatment. St. Louis, MO: Quality Medical Publishing, Inc, 2000, 39 - 104.

Atula T, Hedström J, Finne P, et al (2003). Tenascin-C expression and its prognostic significance in oral and pharyngeal squamous cell carcinoma. Anticancer Res, 23, 3051-6.

Boerner SL, Asa SL, Biopsy interpretation of the thyroid. Philadelphia, PA: Lippincott Williams \& Wilkins. 2009.

Brahma B1, Yulian ED, Ramli M, et al (2013). Surgical perspective of T1799A BRAF mutation diagnostic value in papillary thyroid carcinoma. Asian Pac J Cancer Prev, 14, 31-7

Buchou T, Vernet M, Blond O, et al (2003). Disruption of the regulatory beta subunit of protein kinase CK2 in mice leads to a cell-autonomous defect and early embryonic lethality. Mol Cell Biol, 23, 908-15.

Dominguez I, Sonenshein GE, Seldin DC (2009). Protein kinase 


\section{Miao Guo et al}

CK2 in health and disease: CK2 and its role in Wnt and NFkappaB signaling: linking development and cancer. Cell Mol Life Sci, 66, 1850-7.

Duncan JS, Litchfield DW (2008). Too much of a good thing: the role of protein kinase CK2 in tumorigenesis and prospects for therapeutic inhibition of CK2. Biochim Biophys Acta, 1784, 33-47.

Faust RA, Gapany M, Tristani P, et al (1996). Elevated protein kinase CK2 activity in chromatin of head and neck tumors: association with malignant transformation. Cancer Lett, 101, 31-5.

Giusiano S, Cochet C, Filhol O, et al (2011). Protein kinase CK2alpha subuit over-expression correlates with metastatic risk in breast carcinomas: quantitative immunohistochemistry in tissue microarrays. Eur J Cancer, 47, 792-801.

Guerra B (2006). Protein kinase CK2 subunits are positive regulators of AKT kinase. Int J Oncol , 28, 685-93.

Guo C, Yu S, Davis AT, Ahmed K (1999). Nuclear matrix targeting of the protein kinase CK2 signal as a common downstream response to androgen or growth factor stimulation of prostate cancer cells. Cancer Res, 59, 1146-51.

Howlader N (2012). SEER Cancer Statistics Review 1975-2009 (Vintage 2009 Populations).

Jemal A, Bray F, Center MM, et al (2011). Global cancer statistics. CA Cancer J Clin, 61, 69-90.

Kim JS, Eom JI, et al (2007). Protein kinase CK2alpha as an unfavorable prognostic marker and novel therapeutic target in acute myeloid leukemia. Clin Cancer Res, 13, 1019-28.

Koperek O, Prinz A, et al (2009). Tenascin C in medullary thyroid microcarcinoma and C-cell hyperplasia. Virchows Arch, 455, 43-8.

Landesman-Bollag E, Song DH, et al (2001). Protein kinase CK2: signaling and tumorigenesis in the mammary gland. Mol Cell Biochem, 227, 153-65.

Laramas M, Pasquier D, Filhol O, et al (2007). Nuclear localization of protein kinase CK2 catalytic subunit (CK2alpha) is associated with poor prognostic factors in human prostate cancer. Eur J Cancer, 43, 928-34.

Leins A, Riva P, Lindstedt R, et al (2003). Expression of tenascin-C in various human brain tumors and its relevance for survival in patients with astrocytoma. Cancer, 98, 2430-9.

Lin KY, Tai C, Hsu JC, et al (2011). Overexpression of nuclear protein kinase CK2 alpha catalytic subunit (CK2alpha) as a poor prognosticator in human colorectal cancer. PLoS One, 6, 17193.

Litchfield DW (2003). Protein kinase CK2: structure, regulation and role in cellular decisions of life and death. Biochem $J$, 369, $1-15$.

Lyons AJ, Jones J (2007). Cell adhesion molecules, the extracellular matrix and oral squamous carcinoma. Int $J$ Oral Maxillofac Surg, 36, 671-9.

Mackie EJ, Chiquet-Ehrismann R, Pearson CA, et al (1987). Tenascin is a stromal marker for epithelial malignancy in the mammary gland. Proc Natl Acad Sci USA, 84, 4621-5.

Midwood KS, Hussenet T, Langlois B, Orend G. (2011). Advances in tenascin-C biology. Cell Mol Life Sci, 68, 3175-99.

Oskarsson T, Acharyya S, Zhang XH, et al (2011). Breast cancer cells produce tenascin $\mathrm{C}$ as a metastatic niche component to colonize the lungs. Nat Med, 17, 867-874.

O-charoenrat P, Rusch V, Talbot SG, et al (2004). Casein kinase II alpha subunit and $\mathrm{C} 1$-inhibitor are independent predictors of outcome in patients with squamous cell carcinoma of the lung. Clin Cancer Res, 10, 5792-5803.

Ranjbari N, Almasi S, Mohammadi-Asl J, Rahim F (2013). BRAF mutations in Iranian patients with papillary thyroid carcinoma. Asian Pac J Cancer Prev, 14, 2521-3

Seldin DC, Lou DY, et al (2008). Gene targeting of CK2 catalytic subunits. Mol Cell Biochem, 316, 141-7.

Stalter G, Siemer S, Becht E, et al (1994). Asymmetric expression of protein kinase CK2 subunits in human kidney tumors. Biochem Biophys Res Commun, 202, 141-7.

Tseleni-Balafouta S, Gakiopoulou H, Fanourakis G, et al (2006). Tenascin-C protein expression and mRNA splice variants in thyroid carcinoma. Exp Mol Pathol, 80, 177-82.

Xiao J, Liu C, Hou J, et al (2011). Ser149 is another potential PKA phosphorylation target of Cdc25B in G2/M transition of fertilized mouse eggs. J Biol Chem , 286, 10356-66.

Zhang S, Long H, Yang YL, et al (2013). Inhibition of CK2alpha down-regulates Notch1 signalling in lung cancer cells. J Cell Mol Med, 17, 854-62. 